\title{
Graphene Foam: Hole-Flake Network for Uniaxial Supercompression and Recovery Behavior
}

\author{
Douxing Pan, $*, \ddagger$, Chao Wang, ${ }^{\ddagger}$ and Xiaojie Wang ${ }^{* \dagger}$ \\ ${ }^{\dagger}$ Bio-inspired Robotics and Intelligent Material Laboratory, Institute of Advanced Manufacturing Technology, Hefei Institutes of \\ Physical Science, Chinese Academy of Sciences, Changzhou 213164, China \\ ${ }^{\ddagger}$ The State Key Laboratory of Nonlinear Mechanics (LNM), Institute of Mechanics, Chinese Academy of Sciences, Beijing 100190, \\ China
}

Supporting Information

ABSTRACT: We employed the coarse-grained molecular dynamics simulation method to systematically study the uniaxial supercompression and recovery behavior of multiporous graphene foam, in which a mesoscopic threedimensional network with hole-graphene flakes was proposed. The network model not only considers the physical cross-links and interlayer van der Waals interactions, but also introduces a hole in the flake to approach the imperfection of pristine graphene and the hierarchical porous configuration
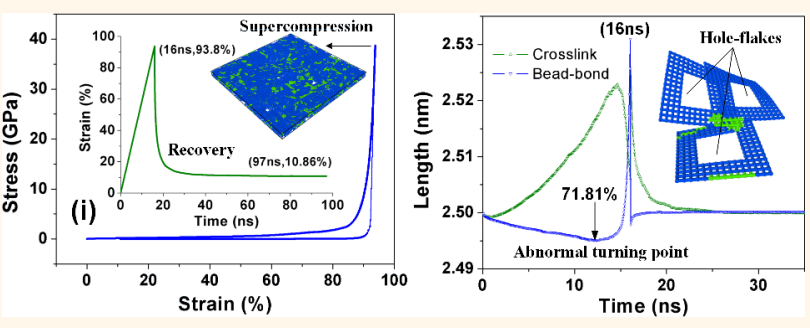

of real foam material. We first recreated a typical two-stage supercompression stress-strain relationship and the corresponding time-dependent recovery as well as a U-type nominal Poisson ratio. Then the recovery unloading at different strains and multicycle compression-uncompression were both conducted; the initial elastic moduli in the multicycles were found to be the same, and a multilevel residual strain was disclosed. Importantly, the residual strain is not exactly the plastic one, part of which can resurrect in the subsequent loading-unloading-holding. The mesoscopic mechanism of viscoelastic and residual deformation for the recovery can be attributed to the van der Waals repulsion and mechanical interlocking among the hole-flakes; interestingly, the local tensile stress was observed in the virial stress distribution. Particularly, an abnormal turning point in the length-time curve for the mean bead-bond length was captured during the supercompression. After the point, the length abnormally increases for different size ratios of the hole to the flake, which is in line with the mesostructure evolution. The finding may provide a mesoscopic criterion for the supercompression of graphene foam related materials.

KEYWORDS: coarse-grained molecular dynamics, graphene foam, supercompression, recovery behavior, strain history, mesoscale, van der Waals interaction, viscoelastoplasticity

$\mathrm{G}$ raphene foam (GF) is a three-dimensional (3D) interconnected macro- and meso-, and sometimes microporous structural material consisting of sheetlike nanocarbon building blocks mainly in an orderless fashion, which could be created using mono/bi/multilayered graphene, belonging to a typical type of carbon foam. ${ }^{1-4}$ In the laboratory condition, the apparent density of GF can be low down to $0.16 \mathrm{mg} / \mathrm{cm}^{3},{ }^{5}$ and high up to $1580 \mathrm{mg} / \mathrm{cm}^{3}{ }^{36}$ The specific area of chemically cross-linked graphene oxide aerogels is as large as $850 \mathrm{~m}^{2} / \mathrm{g}$, and the specific energy adsorption is as much as $45 \mathrm{~J} / \mathrm{g}^{7}$. The naturally dried GF possesses both high electrical conductivities of $1.3 \mathrm{~S} / \mathrm{cm}$ and low thermal conductance of $0.018 \mathrm{~W} /(\mathrm{mK}){ }^{8,9}$ Due to the combined advantages of multiporous materials and two-dimensional (2D) graphene sheets, superior mechanical properties of 3D GFs have received much attention. For example, the foam can sustain structural integrity under a load of over 50000 times its own weight and rapidly recover from over $80 \%$ compression through mimicking the hierarchical structure. ${ }^{10}$ It exhibits a large range of tunable Poisson ratio behavior of $-0.3<\nu<$ 0.46 by simply adjusting the prefreeze temperature. ${ }^{8}$ Because the GF combines both cork-like and rubber-like properties, it can recover up to $98 \%$ compression in air and $90 \%$ in liquids. ${ }^{11}$ An approximate quadratic dependence of compression modulus $E_{\mathrm{c}}$ on foam's density $\rho$ has been obtained..$^{10,11}$ The energy dissipation of foam material was found to be highly stable, as the loss tangent values are retained for as high as 50000 cycles. $^{12}$ The storage and loss moduli as well as

Received: August 28, 2018

Accepted: October 31, 2018 

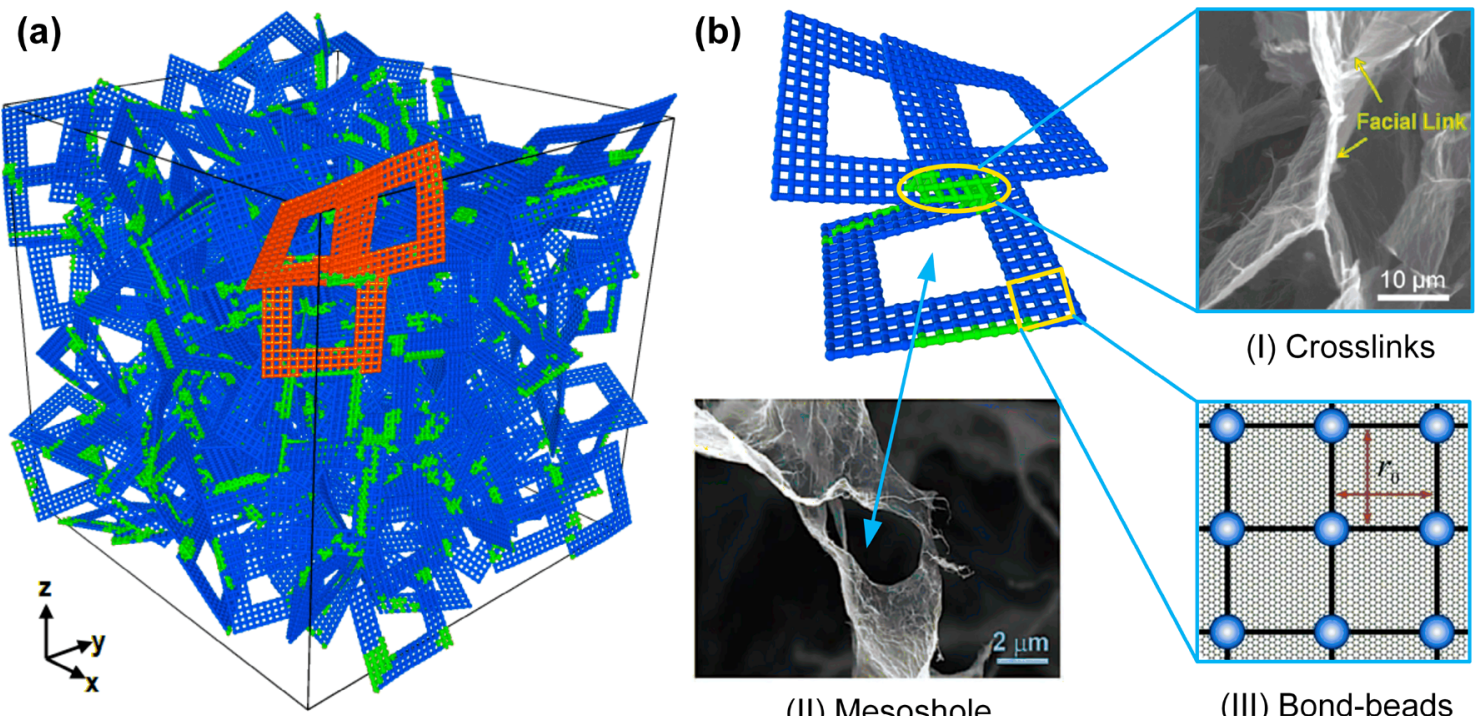

(II) Mesoshole

(III) Bond-beads

Figure 1. Schematic of three-dimensional (3D) hole-flake network and its local structures for 3D multiporous graphene foams (GFs). (a) Relaxed configuration of the 3D numerical sample with physical cross-links (in green) and hole-flakes (in blue). (b) Three mesoscopic twodimensional (2D) linked hole-graphene flakes: (I) SEM image ${ }^{33}$ for physical cross-links; (II) SEM image ${ }^{34}$ for intrinsic holes in graphene flakes; (III) nine bond-beads representative of an eight-layer graphene sheet. ${ }^{26}$

damping constant are insensitive to ambient temperature and loading frequency. ${ }^{13}$ In situ indentation of the 3D graphene framework revealed an extraordinary spring constant of $\sim 15$ $\mathrm{N} / \mathrm{m}$ and an upward of $70 \%$ recoverable deformation. ${ }^{14} \mathrm{~A}$ series of tensile tests were carried out to evaluate the tensile properties and corresponding multiscale deformation mechanisms of free-standing GF and GF-based materials, ${ }^{15-17}$ and a multipeak tensile relationship and a ductile fracture mode were revealed. ${ }^{17}$ Moreover, the study of Krueger et al. ${ }^{18}$ showed the growth and differentiation of $\mathrm{C} 2 \mathrm{C} 12$ myoblasts into functional myotubes on GF bioscaffolds and a subsequent localized substrate movement of over $100 \mu \mathrm{m}$. The biocompatibility of GF scaffold was demonstrated by Nieto et al. ${ }^{19}$ via culturing of human mesenchymal stem cells for a robust proliferation period of 28 days. Whereas there has been extensive experimental investigation of the mechanical properties and/ or behaviors of GF, there has been less theoretical/computational work on $2 \mathrm{D}$ graphene blocks having $3 \mathrm{D}$ connectivity or 3 interactions, or both. Until 2014/2015, Baimova et al. ${ }^{20,21}$ reviewed and researched the nanostructures and mechanical properties of bulk amorphous carbon materials subject to the shear deformation and hydrostatic compression based on allatom molecular dynamic simulations (AAMD). Later, Wang et $a^{22,23}$ systematically applied coarse-grained molecular dynamic simulations (CGMD) to reproduce the rubber-like stressstrain curve in uniaxial compression and dissipative energy variation in cyclic compression-tension. They proposed a tunable Poisson ratio via the stiffness of GF flakes, and uncovered the sliding, impacting, and rippling of graphene sheets as three major energy dissipation mechanisms. Qin et al. ${ }^{24}$ combined AAMD with 3D-printed models showing the 3D graphene assembly having an ultimate tensile strength of 10 times as strong as mild steel at a density of $4.6 \%$ relative to the latter. In line with Nieto et al.'s investigation, ${ }^{19}$ Pan et al. ${ }^{25}$ further examined the mesostructure evolution of tensile behavior of 3D GF expanded from a 2D graphene mesoscopic model $^{26}$ via simply parametrized physical cross-links. Their simulating results clearly demonstrate the squeezing and unsticking process of graphene flakes, the appearance and disappearance of rippling and fluttering flakes, and the breakage of bonds and cross-links. On the basis of the CGMD model for a 2D graphene sheet established by Ruiz et al., ${ }^{27}$ Shen et al. ${ }^{28}$ found the size of graphene sheets playing an important role in both the structural and mechanical properties of GF. By the same model, Xia et al. ${ }^{29}$ found bulk graphene materials in their glassy foam state have an exceptionally large free-volume and high thermal stability as compared to conventional polymer materials. However, all of these computational models are rather ideal, in which all of the graphene flakes are very perfect, and the hierarchical porous configuration of real foam material cannot be embodied at all, far from the experiments. ${ }^{1-4}$ This issue is fairly important because the geometrical morphology of foam skeleton is directly related to the distribution and transfer of local stress. On the other hand, although there are many supercompression and uncompression tests, ${ }^{5,8-11}$ the concept and mechanism of supercompression are not clear, theoretically. In particular, the time-dependent recovery has not been reported yet, systematically. For example, which compressive strain is defined as the supercompressive strain, $70 \%, 80 \%$, or $90 \%$ ? What is the mesoscopic criterion of supercompression? To fully understand the time-dependent recovery behavior, we have to know the strain history, because the strain-time curve can disclose the elasticity, plasticity, and viscosity of GF material to a large extent. $^{30,31}$ Unfortunately, such an important unloading history of the supercompression is not seen in either computations, or experiments.

In this work, a mesoscopic 3D hole-flake network (Figure 1a) was designed to systematically evaluate and predict the supercompression and time-dependent recovery behavior of multiporous GF, based on the CGMD simulations. The network model not only considers the physical cross-links (Figure 1b-I) and van der Waals (vdW) forces between interlayers, but also introduces an intrinsic hole in the graphene flake (Figure 1b-II) to approach the defective graphene sheet in the real GF, and reflect the fabricability of 

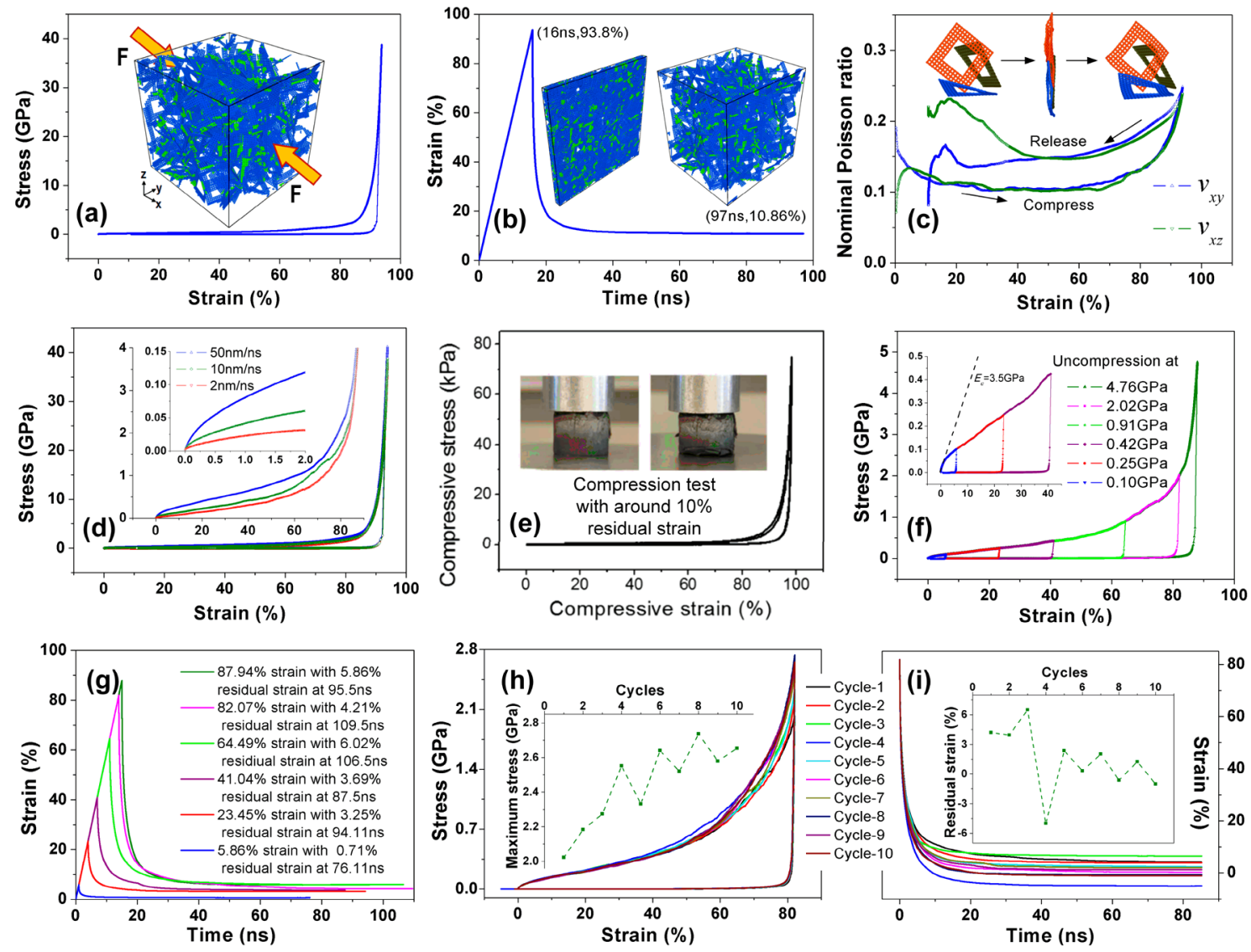

Figure 2. Uniaxial supercompression and uncompression behaviors of GF. (a) Stress-strain curve, the inset is the initial loading state of sample. (b) Strain-time curve, the insets are the deformation states in the largest loading and unloading times, respectively. (c) Change of nominal Poisson ratio with strain, the insets are the mesoscopic structures in three stages corresponding to the insets in (a, b). (d) Ratedependent effect of the supercompression. (e) Supercompression-uncompression testing curve reprinted from Qiu et al..$^{5}$ (f, g) Unicyclic compression-uncompression and corresponding strain-time curves at different unloading stresses or strains, respectively. The dashed line in (f) indicates the slope of proportional elastic stage, i.e., the modulus of compressive elasticity of $3.5 \mathrm{GPa}$. (h, i) Multicycle compressionuncompression and corresponding strain-time curves at the strain of $82.07 \%$, respectively. The insets are the variations of maximum stress and residual strain in cycles, respectively. The dynamic process of supercompression and uncompression is shown in Movie S1 in Supporting Information.

pristine foam skeleton. By means of the proposed network, we not only uncovered the intrinsic mechanical characteristics of supercompression and uncompression in the experiments, but also predicted some interesting results, such as a multilevel residual strain, the same initial elastic modulus, and a hole-flake size ratio-dependent mechanical response. The mesoscopic evolution process and mechanism, the local virial stress distribution, as well as the deformation and energy trends of bond/cross-link/angle were all investigated systematically. We hope that this study could provide an effective way to understand the mesoscopic physical and mechanical nature of 3D GFs, and contribute to an optimal design of advanced GF materials for future applications.

\section{RESULTS AND DISCUSSION}

3D Numerical Network with Intrinsic Holes. Currently, the $3 \mathrm{D}$ GF model is mainly based on the $2 \mathrm{D}$ AAMD or CGMD graphene model through proper 3D treatments. ${ }^{20-29}$ In this work, a 2D graphene mesoscopic model established by Cranford and Buehler is preferentially adopted due to its larger mesoscale (Figure 1b-III). ${ }^{25,26}$ To systematically investigate the mechanical properties and conformational features of $3 \mathrm{D}$ GFs, a huge cubic numerical sample including at least 216 mesoscopic 2D graphene flakes was used as the initial geometrical configuration. Each square flake with length of at $35 \mathrm{~nm}$ was used as the building block as shown in Figure $1 \mathrm{~b}$. One flake in real materials contains 1-10 graphene layers, ${ }^{3,4,17,19}$ so eight layers were adopted here. This can also approach the graphene branch in in situ SEM sample very well, ${ }^{17}$ where the $2 \mathrm{D}$ peak of GF Raman spectrum is 2726.1/ $\mathrm{cm}$, comparable to the peak position of GF used by Chae et al. ${ }^{32}$ indicating $\sim 8-10$ graphene sheets. For simplicity, all the flakes in the numerical sample were assumed to be identical. Each coarse-grain graphene flake was strictly controlled by the $2 \mathrm{D}$ mesoscopic model, and the interlayer (out-of-plane) vdW interaction between particles situated in different neighboring coarse-grain flakes was described as a Lennard-Jones potential

$$
\phi_{\mathrm{op}}=4 \varepsilon_{\mathrm{op}}\left[\left(\sigma_{\mathrm{op}} / r\right)^{12}-\left(\sigma_{\mathrm{op}} / r\right)^{6}\right]
$$

where $\varepsilon_{\mathrm{op}}$ indicates the depth of potential well, $\sigma_{\mathrm{op}}$ is the position of minimum potential, and $r$ is the distance between two beads among interlayer graphene flakes in a cutoff range. The parameters were listed in Table $S 1$ in the Supporting Information, and the cutoff of Lennard-Jones potential was taken as $5 \mathrm{~nm}$, above which the interaction between two particles tends to be insensitive to the distance. ${ }^{25}$ 


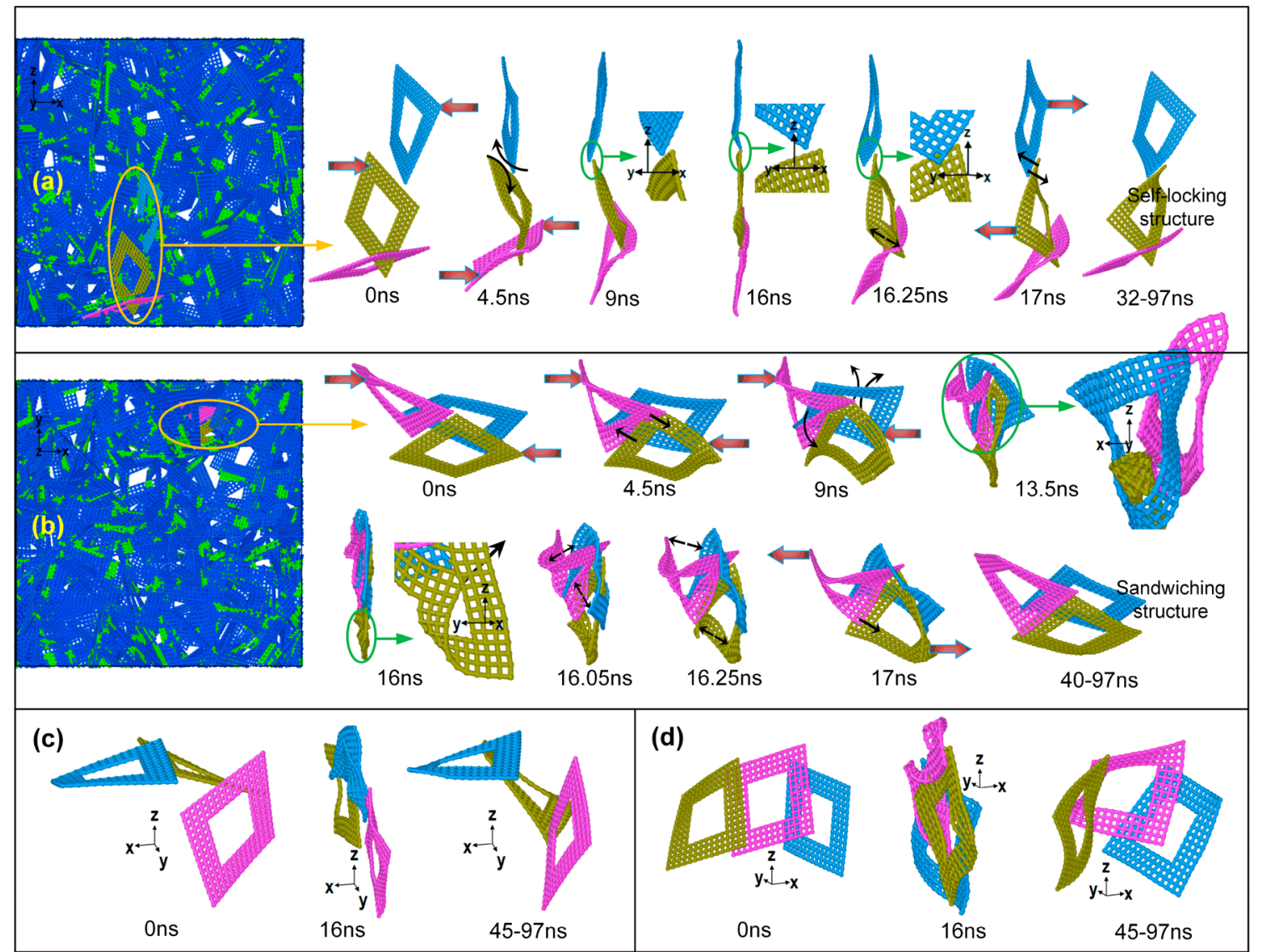

Figure 3. Evolution of mesostructures in supercompression and uncompression. (a) Forming process of self-locking structure in front view along $y$-axis (see also Movie S2 in the Supporting Information). (b) Forming process of the sandwiching structure in front view along $z$-axis (see also Movie S3 in the Supporting Information). (c, d) Two typical hole-induced mechanical interlocking in initial loading, supercompressive, and long holding states, respectively. Three different constituent hole-graphene flakes are labeled in brown, pink, and indigo, respectively. The fat-red and thin-black arrows indicate the external and interior forces, respectively. The detailed drawings for (a) and (b) are in the corresponding local coordinate systems. The status is in the compression process before $16 \mathrm{~ns}$, and it is in the uncompression process after $16 \mathrm{~ns}$.

During the actual manufacturing process of GFs, due to the squeezing effect or covalent attachment of functional groups, the graphene sheets would form a monolithic chemically or physically linked 3D network. ${ }^{10,11,33}$ On the basis of this fact, 3D linkages were preconsidered in the numerical GF sample before loading/unloading, as labeled with green sticks in Figure la,b-I, ${ }^{33}$ and a cross-link model with harmonic spring potential

$$
\phi_{\mathrm{cl}}=k_{\mathrm{cl}}\left(r-r_{\mathrm{cl}}\right)^{2} / 2
$$

was used to describe the $3 \mathrm{D}$ cross-links among different flakes, where $k_{\mathrm{cl}}$ is the spring constant and $r$ is the distance between two beads situated in different neighboring coarse-grain flakes with a referenced equilibrium distance $r_{\mathrm{cl}}$. The corresponding parameters were set to be the same as those for the $2 \mathrm{D}$ beadbond listed in Table S1, which were proved to be well with our previous work for the tensile deformation. ${ }^{25}$

The real graphene flake is not usually perfect, as shown in Figure 1b-II, so an intrinsic hole in the graphene flake was introduced to represent the imperfection. ${ }^{5,34}$ On the other hand, physically, the electronic structures are very different between the perfect and imperfect graphene, which would directly lead to different electromechanical coupling effects. $^{35-39}$ Such a hole-flake can also reflect the hierarchical configuration and fabricability of pristine GF skeleton, ${ }^{2}$ which are closely related to the $3 \mathrm{D}$ connectivity in GF (Figure 1). In order to describe the influence of intrinsic hole to the GF, a ratio of the size of hole to flake is defined as

$$
\kappa=\left(L^{\mathrm{h}} \times W^{\mathrm{h}}\right) /\left(L^{\mathrm{f}} \times W^{\mathrm{f}}\right)
$$

where $L^{\mathrm{h}}$ and $W^{\mathrm{h}}$ are the length and width of hole, respectively, and $L^{\mathrm{f}}$ and $W^{f}$ are the length and width of whole flake, respectively. As will be shown in what follows, different holeflake ratios can lead to different mechanical responses of GFs.

Two-Stage Supercompression and Time-Dependent Uncompression. Figure 2a shows a typical supercompression-uncompression stress-strain curve, and reasonably reproduced the experiment in Figure 1e, where the GF also consists of mesoscopic pristine hole-graphene sheets. ${ }^{5}$ Such a cyclic stress-strain curve is one of the most common mechanical behaviors in the supercompression-uncompression, ${ }^{10,11}$ usually seen in other GF materials with different structures, such as vortex, laminated, and hyperbolic configurations. $^{8,9}$ Figure $2 \mathrm{~b}$ shows the corresponding straintime curve, and the compressive strain has reached $93.8 \%$ when the stress is released at $16 \mathrm{~ns}$, and then the strain decreases until $60 \mathrm{~ns}$, with a residual strain of $10.86 \%$ retained at $97 \mathrm{~ns}$. The recovery is time-dependent, meaning an obvious viscoelastic behavior before $60 \mathrm{~ns}$. In the small-strain mechanical tests, GF and GF-based composites have been verified as viscoelastic materials by means of the storage and 
loss moduli as well as damping constant. ${ }^{12,13,40}$ The insets in Figure $2 \mathrm{~b}$ are the deformation states of GF in the largest loading and unloading times, respectively. The latter is different from the initial state presented in the inset of Figure 2a, meaning an irreversible deformation, ${ }^{25,40,41}$ which is almost unchanged from 60 to $97 \mathrm{~ns}$. In the experiment of Figure 2e, there is also a residual strain of $10 \%$ around, and in the second inset an obvious yield deformation can be observed. ${ }^{5}$ The dynamic process of supercompression-uncompression is shown in Movie S1 in the Supporting Information. Figure 2c shows a U-type nominal Poisson ratio curve ${ }^{9}$ with strain for both the compression and uncompression, which mainly originates from the rearrangement of graphene flakes as shown in the insets corresponding to the three deformation states of insets in Figure 2a,b. It has been reported in our previous work for the GF without physical cross-links and intrinsic-holes, ${ }^{22}$ and it can be influenced by the hole-flake ratio, as shown in Figure $5 c$,f. In order to further reveal the loading rate feature of viscoelasticity, different deformation rates were carried out for the same sample, and the results are presented in Figure $2 \mathrm{~d}$. The GF deforms rate-dependently, and different loading curves ramify with different initial elastic region, and gather in a supercompressive stress of $4.0 \mathrm{GPa}$ in the strain of $\sim 87 \%$. This can be mainly attributed to the intrinsic rate-dependent response of mesoscopic structures in the GF to the external loading, as usually explained for the uniaxial mechanical behaviors in polymers such as polycarbonate ${ }^{31}$ and poly(ether imide). ${ }^{41}$ In Figure 2, the compression behavior contains a slowly and a quickly increased stress stages, and the strain of transition region is $\sim 75 \%$, over which it is phenomenologically thought to be the supercompression, until a limit of compressive strain (Figure S1).

Figure $2 \mathrm{f}$ shows a group of unicyclic compression-recovery unloading at different stress levels from the same supercompression curve. The corresponding strain-time curves are shown in Figure 2g, presenting a bunch of typical exponential viscoelastic recoveries with multilevel asymptotic lines. The compression-uncompression loops with various stress levels were usually reported in the GF experiments, and varied with GF configurations. ${ }^{9,42,43}$ This interesting multilevel residual strain can be directly attributed to the mechanical interlocking, which would be revealed by the evolution of mesoscopic structures in the following. Figure $2 \mathrm{~h}, \mathrm{i}$ show a multicycle supercompression-uncompression with maximum strain of $82.07 \%$ and the corresponding recovery curve family, respectively. Likewise, a multilevel residual deformation is presented in Figure 2i. The insets in Figure 2h,i are the variations of maximum stress and residual strain in the cycles, respectively. The former has an ascend trend in total, while the latter has no such obvious trend. The former is closely related to the total amount of deformation (Figure S2), while the latter is closely related to the mesoscopic evolution that will be further uncovered. The loading-unloading loops presented in Figure $2 \mathrm{~h}$ are very different from the compression-tension ones, ${ }^{34}$ where the minimal and maximal strain are both controlled by the external deformation with a compression and tension speed of $600 \mathrm{~mm} / \mathrm{min}$. The compression-tension loop cannot reflect the self-relaxation in the full recovery, although it partly performs the energy dissipation form and corresponding mechanism of GF. ${ }^{23}$ It is noted that the initial compressive moduli in Figure $2 \mathrm{~h}$ are the same for the different loops, i.e., $E_{c}$ $=3.5 \mathrm{GPa}$ (Figure $2 \mathrm{f}$ ) without softening or hardening. This implies that the multicycle loading and unloading for the supercompression cannot alter the intrinsic elastic properties of $\mathrm{GF}$, which is mainly decided by the holed graphene-flake itself. ${ }^{4,36,44}$

Evolution of Mesostructure and Virial Stress Distribution. Figure 3 shows the evolution of mesostructures in the supercompression and uncompression, and in the initial loading time of Figure 3a, the three hole-flakes retain their freestanding states. The indigo flake keeps away from the brown flake, and the cross-links between the brown and pink flakes are in the energy minimization. As the compression increases, three flakes deform, then the indigo and brown flakes come into the scope of vdW interaction, and the cross-links distort under the stress from the unbalance of pink and brown flakes. At $4.5 \mathrm{~ns}$, the indigo and brown flakes get very close in both the vdW attraction and squeezing from other flakes, so that they start to repel in a larger vdW repulsion. Due to the pull of pink flake, the brown flake slides down along the indigo one, as indicated by the two thin-black curved arrows, and the corresponding friction contributes to the viscosity. ${ }^{23}$ Under the $\mathrm{vdW}$ repulsion and pulling of pink flake, the brown flake slides over the indigo one at $9 \mathrm{~ns}$, as shown in the detail drawing. At $16 \mathrm{~ns}$, the brown flake suffers a huge internal force from the pink flake and serious squeezing from other flakes around, and the indigo flake is repelled up by the brown one along the $z$ axis. Due to the supercompression, the pink and brown flakes are pressed together tightly, so are other flakes. As a result, a huge resilience would make the flakes get back to shape as much and quickly as possible when the external force is removed, as shown at $16.25 \mathrm{~ns}$ in Figure $3 \mathrm{a}$, where the brown flake is powerfully pushed away from the pink one, as labeled by the two arrows. At the same time, the brown flake slides into the right side of indigo one, as shown in the detail drawing of $16.25 \mathrm{~ns}$. Such a selective right-sliding is mainly decided by the magnitude and direction of local resilience and the morphology and slope of potential barrier provided by the indigo flake. However, the right-sliding flake cannot return to its original position due to the resisting from the indigo flake as shown in the detail drawing of $17 \mathrm{~ns}$, although the resilience continues to function. From 32 to $97 \mathrm{~ns}$, the dynamic state of three labeled flakes remains almost unchanged, so is that of other flakes. The unrecoverable state of indigo and brown flakes is called mechanical interlocking in mechanics, which is the main reason for residual strain in the GF. Similar interlocking phenomenon has also been reported in the field of bionic mechanics. ${ }^{45}$ The self-lock structure not only appears in the supercompression, but also forms in the general compression, such as the status at 9 ns around with the strain of $\sim 52.76 \%$. This is the main reason for multilevel residual strain in the recovery curves for the unloading at different stress levels from the same supercompression curve (Figure $2 \mathrm{~g}$ ). The deformation degree is determined by the amount of self-lock mesostructures and the occurrence probability of mechanical interlocking in the compression. The Supporting Information provides a Movie S2 for the above evolution process.

Figure $3 \mathrm{~b}$ further shows a forming process of an interesting sandwiching structure. In the initial time, the pink and brown flakes do not contact each other. In the compressive deformation along the $x$-axis, they contact and squeeze as a form of edge-to-edge until they both bend seriously. At $9 \mathrm{~ns}$, they pass through the hole of the indigo flake, and one corner of the latter also passes through the hole of the pink flake. Such a through-hole was also discussed in the experiment, where the 


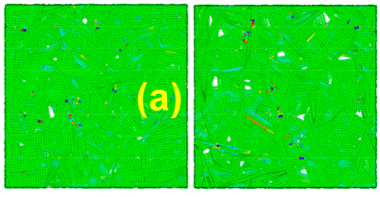

$\left(\varepsilon_{x x}^{m}, \sigma_{x x}^{m}\right)=(0,0)$

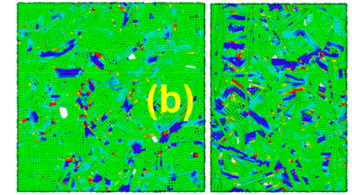

$(25.5 \%, 0.27 \mathrm{GPa})$

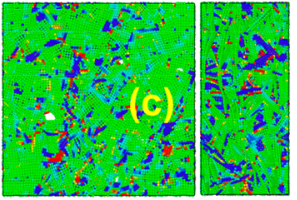

$(51.88 \%, 0.55 \mathrm{GPa})$
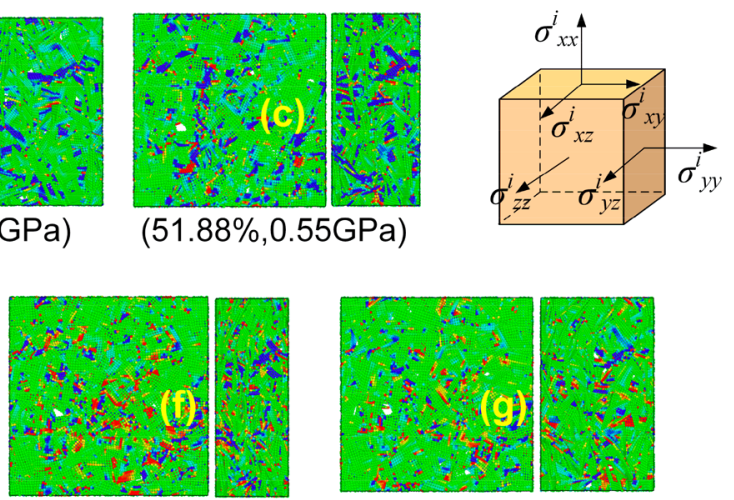

$(60.2 \%, 0.005 \mathrm{GPa})$

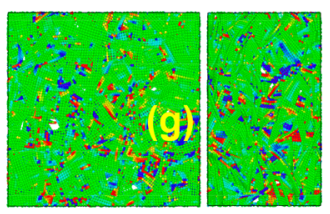

$(37.71 \%, 0.0008 \mathrm{GPa})$

$(78.26 \%, 1.59 \mathrm{GPa})$

(93.51\%,33.48GPa)

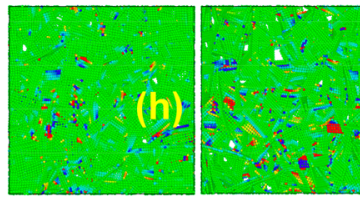

(12.24\%,2.01e-5GPa)

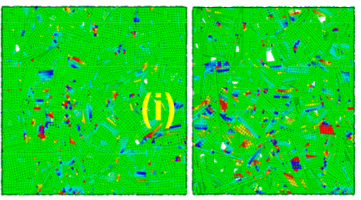

$(11.22 \%, 0 \mathrm{GPa})$

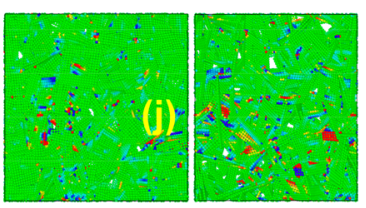

$(10.86 \%, 0 \mathrm{GPa})$
$1.5 \mathrm{e} 9$

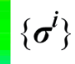

$-1.5 e 9$
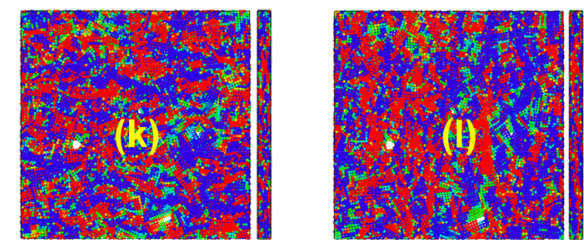

$\left(\varepsilon_{y y}^{m}, \sigma_{y y}^{m}\right)=(-22.5 \%, 0) \quad\left(\varepsilon_{z z}^{m}, \sigma_{z z}^{m}\right)=(-21.8 \%, 0)$

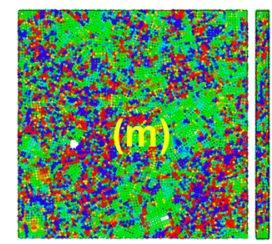

$\left(\varepsilon^{m}{ }_{x y}, \sigma_{x y}^{m}\right)=(0,0)$

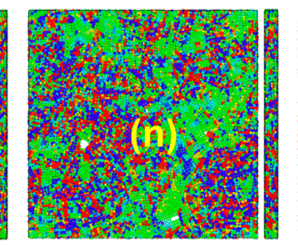

$\left(\varepsilon^{m}{ }_{x z}, \sigma^{m}{ }_{x z}\right)=(0,0)$

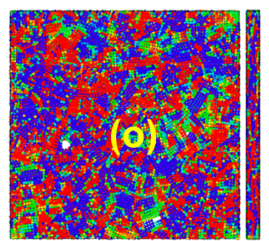

$\left(\varepsilon^{m}{ }_{y z}, \sigma_{y z}^{m}\right)=(0,0)$

Figure 4. Local stress distribution for the GF in supercompression and uncompression. (a-e) Distribution of normal stresses in yoz-plane in different compressive strains. $(f-j)$ Distribution of normal stresses in yoz-plane in different recovery strains. (k-o) Distribution of normal stresses in $x \mathbf{o z}-/ x \mathrm{o} y$-plane and shear stresses in $y o z-/ x \mathrm{o} z-/ x \mathrm{y} y$-plane with strain of $93.51 \%$ in supercompression, respectively. The schematic diagram of stress analytical unit in the GF sample during the compressive deformation is shown on the top-right corner, and the forward directions of normal stresses $\sigma_{x x}, \sigma_{y y}$ and $\sigma_{z z}$ are the position directions of $x$-/y-/z-axis in Cartesian coordinate system, respectively. Every figure includes the top view (compressible direction) on the left and side view on the right.

graphene nanopore was designed to solve the deoxyribonucleic acid sequencing problem. ${ }^{46}$ At $13.5 \mathrm{~ns}$, the indigo and pink flakes wrap each other due to the continuing compressive force, and at $16 \mathrm{~ns}$ even the corner of the brown flake crosses its own hole in the supercompression, as shown in the corresponding detail drawings. Once the external force is removed, the three flakes would get back to shape as much and as quickly as possible due to the huge vdW repulsion, as shown at 16.05 or $16.25 \mathrm{~ns}$ in Figure $3 \mathrm{~b}$. As the compressive strain decreases, the local resilience varies from the repulsion to the pull force among the flakes by means of the cross-links as labeled by the fat red arrows, so that the squeezing and resulting rippling fade away. However, the pink and brown flakes cannot precisely move to their initial positions, due to the diversity of potential path. The brown flake inserts into the gap between the pink and indigo flakes to form a sandwiching structure, as shown at $17 \mathrm{~ns}$. Such a sandwiching structure is rather stable and difficult to be unlocked by the decreasing resilience due to the mesoscopic friction and clamping force, from 40 to $97 \mathrm{~ns}$, as shown in Movie S3 in the Supporting Information. It can be seen from Figure $3 b$ that the intrinsic hole provides enough deformation for the hole-flakes to shrink, but it cannot confine them to spread in removal of the external force. It also plays an important role in the mechanical interlocking, as shown in Figure 3c,d. In Figure 3c, the brown flake passes through the indigo one, and the edge resistance of hole is the main factor of self-lock structure. In Figure 3d, the pink flake passes through the indigo one, and the surface friction is the main factor. Due to the fact that the edge contact force is small, the self-lock structure in Figure $3 \mathrm{c}$ is not very stable and easily unlocked in the multicycle supercompression and uncompression. That is why part of the residual strain is rebound to the viscoelasticity in the subsequent cycles, especially the cycle- 4 and cycle-10, as presented in Figure $2 \mathrm{~h}, \mathrm{i}$. Such a transition from the residual to viscoelastic strains can be also observed in the case of absorption of solvent. ${ }^{47}$ Therefore, the residual strain in the GF is not exactly a plastic behavior that is irreversible in permanent. ${ }^{31,40,41}$

Figure 4 shows the virial stress distribution of uncompressive or compressive GF system. In comparison to the undeformed status, the local compressive stress $\sigma_{x x}^{i}$ has an obvious distribution (blue area) in the compressive strain, as shown in Figure $4 b-d$. Due to the graphene skeleton's formal geometry governed by its connectivity, ${ }^{25,48}$ the stress of GF presents a discretized distribution, which is also observed in the tensile deformation. ${ }^{25}$ Only in the supercompression, the local stress almost distributes everywhere, as shown in Figure $4 \mathrm{e}$. It can be seen from Figure $4 \mathrm{~b}-\mathrm{e}$ that some location seems to be in a self-equilibrium state, i.e., almost no normal compressive stress (green area). This is because the GF consists of the graphene sheets, and the in-plane stress is the main load way. When the normal vector of GF flake is parallel 

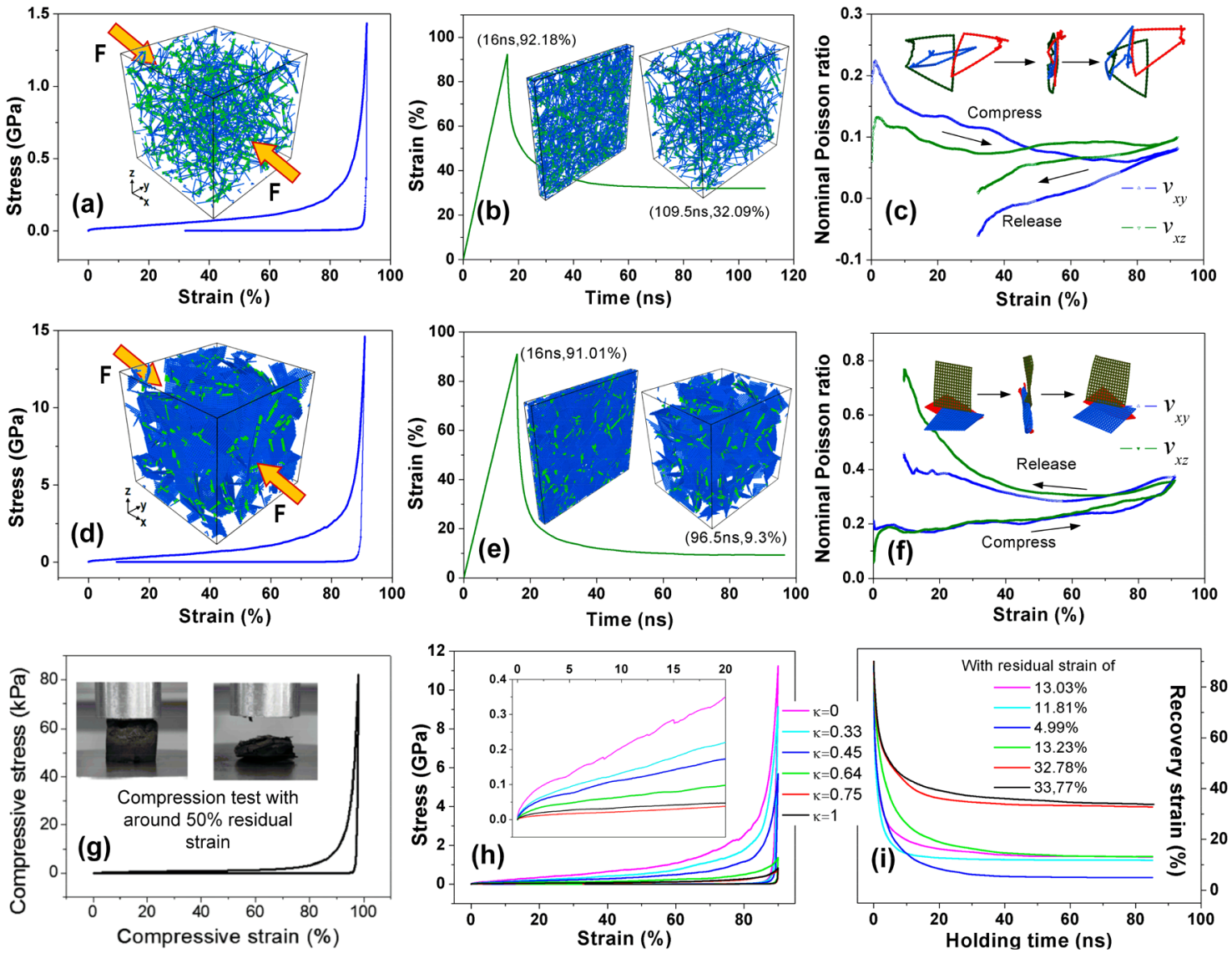

Figure 5. Hole-flake ratio effect of GFs with hole-graphene flakes. (a, d) Stress-strain curves for hole-flake ratios of $\kappa=1$ and $\kappa=0$, respectively; the insets are the initial loading states of two samples, respectively. (b, e) Strain-time curves for $\kappa=1$ and $\kappa=0$, respectively; the insets are the deformation states in the largest loading and unloading time for two samples, respectively. (c, $f$ ) Changes in normal Poisson ratio with strain for $\kappa=1$ and $\kappa=0$, respectively; the insets are the mesoscopic structures in three stages corresponding to the insets in (a, b) and (d, e), respectively. (g) Supercompression-uncompression testing curve reprinted from Qiu et al. ${ }^{5}$ (h, i) Supercompressive stress-strain and corresponding strain-time curves for GFs with different hole-flake ratios, respectively. The dynamic processes of supercompression and uncompression for $\kappa=1$ and $\kappa=0$ are shown in Movie S4 and Movie S5 in the Supporting Information, respectively.

to the $x$-axis, it would not go through the normal compressive stress. This rule is also suitable to the area that shear stresses act on, as shown in Figure $4 \mathrm{~m}-\mathrm{o}$. Some local stress is negative, the other is positive, as shown in Figure $4 \mathrm{k}-\mathrm{o}$ as labeled in blue and red, respectively. This is because the GF flakes in the compression interact intensely on each other through vdW interaction, and most of them are interrelated through the cross-links and holes. The nonsynchronous deformation of randomly distributed flakes leads to the complex hinge force of cross-links, mainly contributing to the local tensile stress among the flakes. Part of flakes passing through the holes also leads to the tensile stress of inside edges of hole-flakes (Figure 3). Even for the normal compressive stress, a weak tensile stress can be also observed in Figure $4 c-e$. The local positive and negative stresses always neutralize except $\sigma_{x x}^{i}$, so that the sum is always zero in agreement with the average stress, i.e.,

$$
\begin{gathered}
\sigma_{h g}^{m}(t)=0=\sum_{i=1}^{I} \sigma_{h g}^{i p}(t)+\sum_{i=1}^{N-I} \sigma_{h g}^{i n}(t) \\
(h=x, y, z ; g=y, z)
\end{gathered}
$$

where $\sigma_{h g}^{i p}$ and $\sigma_{h g}^{\text {in }}$ indicate the local positive and negative stresses, respectively, and $N$ is the number of total particles involved. Moreover, the normal stress $\sigma_{y y}^{i}$ is well-aligned along the $y$-axis (Figure $4 \mathrm{k}$ ), so is $\sigma_{z z}^{i} z$-axis (Figure $4 \mathrm{l}$ ), and the corresponding shear stress $\sigma_{y z}^{i}$ is well-biorthogonal in the yozplane, which mainly contributes to the shearing of flakes being a reason for the elasticity of GF. By contrast, the local shear stresses $\sigma_{x y}^{i}$ and $\sigma_{x z}^{i}$ are very small, which mainly contribute to the slipping between the GF flakes being a reason for the viscosity and plasticity. Figure $4 \mathrm{f}-\mathrm{o}$ show the variation of normal stresses after the uncompression. It can be seen from Figure 4f,g that the blue local compressive stress is much smaller than that in Figure $4 b-e$, but larger than that in Figure $4 \mathrm{a}, \mathrm{h}-\mathrm{j}$, because the viscoelasticity of GF retains the mean compressive stress of 0.005 and $0.008 \mathrm{GPa}$. Due to the local resilience from the vdW repulsion in the uncompression, the red local tensile stress also appears in Figure 4f,g. However, in comparison to Figure $4 \mathrm{a}$, the local stress does not disappear after enough holding time, which is attributed to the mechanical interlocking. An external disturbance is possible to unlock the interlocking. For example, by means of the subsequent cycles, especially cycles of $4,6,8,10$, as shown in Figure $2 \mathrm{~h}, \mathrm{i}$, the self-lock structures formed from the first cyclic loading-unloading-holding can be partially unlocked, especially for the unstable hole-induced self-locks as shown in Figure 3c,d.

Hole-Flake Ratio Effect and Variation of Length/ Angle/Energy. In order to study the influence of intrinsic hole to the GF, different hole-flake ratios were carried out in 

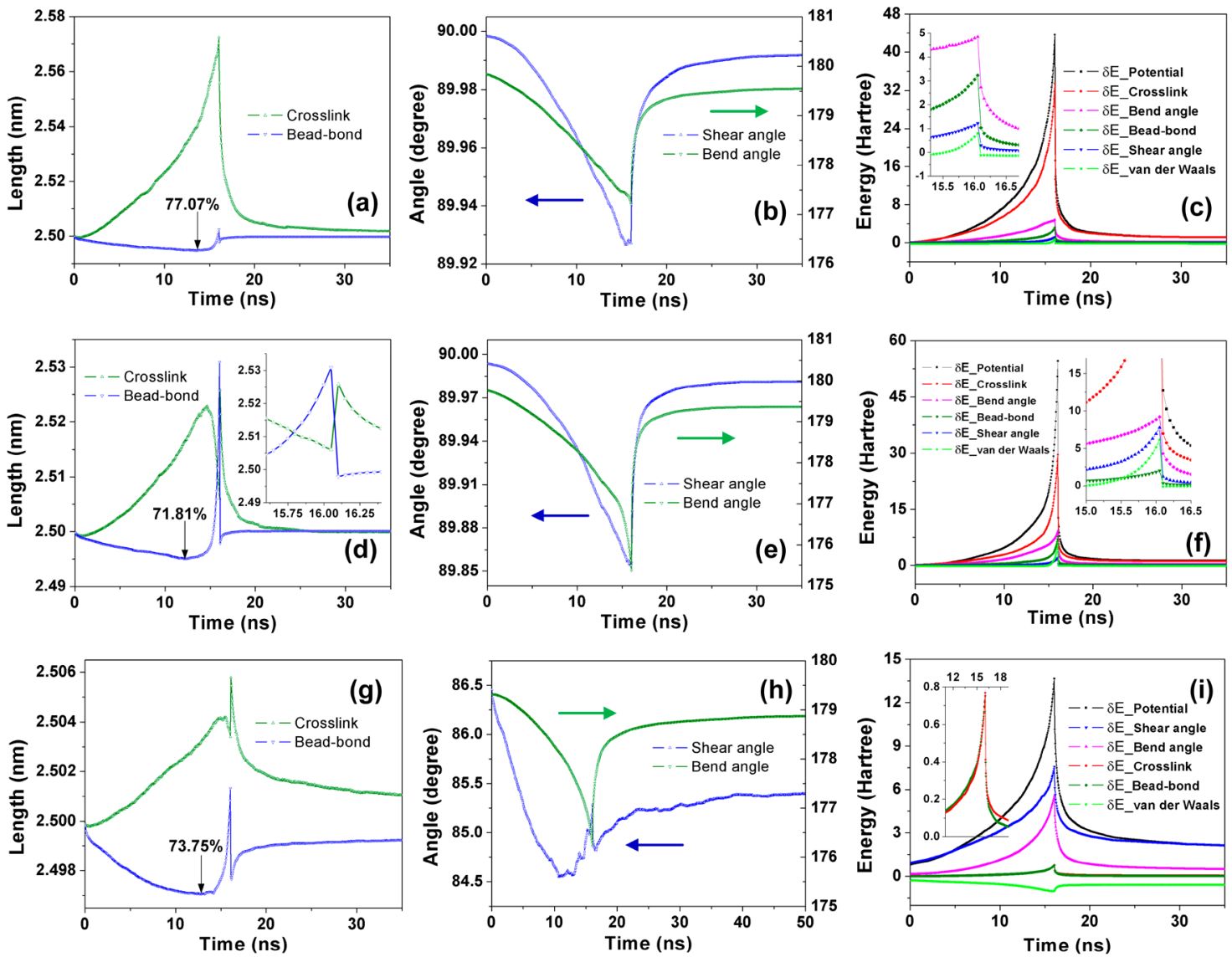

Figure 6. Variation of average bond/angle/energy for three typical GFs with different hole-flake ratios. Variation of average length of beadbond and cross-link with time for (a) $\kappa=0,(\mathrm{~d}) \kappa=0.33$, and $(\mathrm{g}) \kappa=1$, respectively. Average change of shear (left $y$-axis) and bend (right $y$ axis) angles with time for $(\mathrm{b}) \kappa=0,(\mathrm{e}) \kappa=0.33$, and $(\mathrm{h}) \kappa=1$, respectively. Variation of average energy of potential $/ \mathrm{cross}$-link $/ \mathrm{bond} / \mathrm{angle}$ with time for $(\mathrm{c}) \kappa=0$, (f) $\kappa=0.33$, and (i) $\kappa=1$, respectively. The insets in (d, c, f, i) are the magnifying drawings in the middle of horizontal axes.

the supercompression and uncompression. Two limiting cases were first in consideration, i.e., $\kappa=1$ and $\kappa=0$, respectively. For the former, the hole is large enough so that the holegraphene flake can be viewed as a mesoscopic ring, ${ }^{51}$ and the corresponding GF should be viewed as a $3 \mathrm{D}$ graphene-ring network. Figure 5a,b show the stress-strain and strain-time curves for the hole-flake ratio of $\kappa=1$, and the insets are the initial loading state and deformation states in the largest loading and latest unloading times, respectively. Figure 5c shows the variation of nominal Poisson ratio with compressive strain, in which the insets are the local mesoscopic structures in three stages corresponding to the insets in Figure $5 \mathrm{a}, \mathrm{b}$. In comparison to the GF with $\kappa=0.33$ in Figure $2 \mathrm{a}-\mathrm{c}$, the bearing capacity is reduced by an order of magnitude in the supercompressive strain of $92.18 \%$, but the residual strain is increased by 3 times after a long holding time of $80.5 \mathrm{~ns}$, and a negative Poisson ratio is also observed after the uncompression. This is because the ring-like graphene is more flexible to be pressed and easier to get tangled, which is very similar to the polymer chain of poly(ether imide) resin. ${ }^{41}$ The complex network in the supercompression can form a tight structural phase to prevent the resilient deformation in the uncompression, so that the lateral length might be larger than its initial value. For $\kappa=0$, the intrinsic hole disappears and the graphene flake functions as a perfect building block of the GF. Figure $5 \mathrm{~d}$,e show the stress-strain and strain-time curves for this case, and the insets are the initial loading state and deformation states in the largest loading and latest unloading times, respectively. Figure $5 \mathrm{f}$ shows the variation of nominal Poisson ratio with compressive strain, in which the insets are the local mesoscopic structures in three stages corresponding to the insets in Figure 5a,b. In comparison to the GF in Figure 5a, the supercompressive stress in Figure $5 \mathrm{~d}$ is improved by an order of magnitude in the supercompressive strain of $91.01 \%$ smaller than that of the former. Interestingly, although the flake is perfect for $\kappa=0$, the residual strain is still $9.3 \%$, as shown in Figure $5 b$, almost the same as that of the GF with $\kappa=$ 0.33 , as shown in Figure $2 \mathrm{~b}$, after the holding times of 80.5 and $81.0 \mathrm{~ns}$, respectively. Also, the nominal Poisson ratios $v_{x y}$ and $v_{x z}$ are both positive for $\kappa=0.33$ and $\kappa=0$, respectively, whether supercompression or uncompression. It implies that the perfect graphene flake cannot improve the intrinsic mechanical properties of GF, and hence is not appropriate as a target in designing the superelastic GF and GF-based materials. Figure $5 \mathrm{~g}$ shows a stress-strain testing curve in the supercompression and uncompression, ${ }^{5}$ where the residual strain is about $50 \%$, even larger than that in Figure 5a. The intrinsic difference between this experimental sample and that in Figure $2 \mathrm{e}$ is the annealing temperature of freeze-casting postprocessing technique, being 200 and $500{ }^{\circ} \mathrm{C}$, respectively. One of important reasons for the larger elasticity should be the degeneration of self-lock structure in the higher temperature. Figure $5 \mathrm{~h}$ further shows the supercompression curves for different hole-flake ratios unloading at the same strain of $90 \%$. 
It can be seen the GF deforming hole-dependently-the smaller the hole-flake ratio is, the larger the magnitude of stress-strain curve responds. Figure $5 i$ shows the corresponding recovery curves holding to the same time of $85 \mathrm{~ns}$. There is a large gap value of $19.55 \%$ in the multilevel residual strain. The large-hole induced entanglement and self-lock structure jointly lead to the large residual strains in the GF with $\kappa=0.75$ and $\kappa=1$, and the gap should be reduced as the number and degree of entanglements decrease. Additionally, the hole-flake ratio is strongly inversely correlated to the density of GF, thereby the initial elastic modulus, but independent of the shape and symmetry (Table S2).

What is the mesoscopic criterion of supercompression behavior? In refs $5,7-11$, the supercompression is roughly thought to be a state of a larger compressive strain $(>80 \%$ around) with a quick stress increase and without structural collapse. Such a quick increasing stage is also shown by Figures 2,5 , with a transition strain region from 70 to $80 \%$. However, this is only the phenomenological criterion. To further disclose the mesoscopic mechanism of two stages, Figure 6 shows the variations of bond, angle, and energy for three typical GFs, which consist of the perfect-graphene $(\kappa=0)$, hole-flake $(\kappa=$ 0.33 ) and graphene-ring $(\kappa=1)$, respectively. It can be seen clearly that there is an abnormal turning point of the average bead-bond length for all three GFs during the compressing procedure, before which the length regularly decreases as the deformation increases, after which it abnormally increases. The strains of three turning points are $77.07,71.81$, and $73.75 \%$, as shown in Figure 6a,d,g, respectively. On the other hand, the variation trend of average cross-link length seems to be contrary to that of the average bond length (Figure 6a,d,g). The average tension of cross-links mainly derives from the nonsynchronous deformation of randomly distributed flakes (Figure 3 ). The increasing of average bond length is actually caused by the tensile cross-links due to the synergistic effect in the supercompresssion. Therefore, the turning points should be viewed as the mesoscopic criteria of supercompression, after which the average bead-bond length is even larger than its original value as the compression increases (Figure 6a,d,g). A special case is that the variation of average length variation might be caused by several overlong bonds, which can also be included in the scope of criteria. At the moment of unloading, the abnormal tensile energy of bead-bonds delivers to the cross-links, and the bead-bonds revert to the compression state again. There is an obvious jump discontinuity in the lengthtime curve. In this region, the energy exchange is instantaneous, and the corresponding deformation is perfectly elastic. After that, the energies saved in the stretched crosslinks and compressed bead-bonds are released gradually to provide the part of resilience, and then the length tends to its original value. Figure $6 \mathrm{~b}, \mathrm{e}, \mathrm{h}$ show the variation of average shear and bend angles with time, and the variation range of former is much smaller than the latter. This implies the bending of flake playing a leading role in both the supercompression and resilience of recovery. In comparison to Figure $6 \mathrm{~b}, \mathrm{e}$, the variation range of shear angle in Figure $6 \mathrm{~h}$ is much larger, and the trend is nonmonotonic. The reason is that the graphenering is more flexible, and many entanglements are involved. That is also why the ring-type GF has a large residual strain. Figure $6 c, f, i$ provide a group of energy change curves. It can be seen that (i) all energy curves increase in the compression and decrease in the recovery, except the vdW energy curve in Figure 6i, because the ring-type GF has enough space to perform the attractive interaction; (ii) the bending energy in all type GFs is determinative, which derives from the out-of-plane deformation, saved in the supercompression and released in the uncompression to rebound the compressive GF; (iii) for the ring-type GF, the intrinsic hole is the most source of energy saved in the shearing deformation, and for the perfect or general hole-type GF, the cross-link is still the main source of energy saved in the tensile deformation, which contributes to the recovery behavior. In comparison to Figure $6 c$, which possesses a large potential difference between the cross-link and bending angle energies, the energy curves in Figure $6 f, i$ are well-distributed. The reason lies in the fact that the hole-flakes provide more degrees of freedom in the mesoscopic structures (Figure 3), which might lead to a better performance of the hole-type GF in the mechanical properties.

\section{CONCLUSION}

Utilizing intrinsic hole-graphene flakes and physical cross-links, together with consideration of the interlayer vdW interactions, a probably $3 \mathrm{D}$ multiporous graphene assembly was designed to systematically study the uniaxial supercompression and recovery behavior that remain poorly understood, based on the CGMD method. First, we reasonably created a typical twostage supercompression stress-strain relationship and the corresponding time-dependent recovery behavior as well as a U-type nominal Poisson ratio. On this basis, we revealed a multilevel residual strain, the same initial elastic modulus, and a hole-flake ratio-dependent mechanical response. The mesoscopic mechanisms of viscoelastic and residual deformation for the recovery can be attributed to the vdW repulsion and mechanical interlocking among the hole-flakes. Finally, an abnormal turning point was well identified in the length-time curve of mean bead-bond length during the supercompression, which rationally answered what the supercompression is based on the mesoscale for different hole-flake ratios. This study suggests that avoiding/decreasing the self-lock structures should be an important goal in designing the superelastic GF and GF-based composites, and the hole-flake ratio may be a designing factor in tuning the mechanical performance.

\section{METHODS}

2D Mesoscopic Graphene Model. On the basis of the equivalent energy principle and a series of full atomistic calculations of a mechanical test suite, a $2 \mathrm{D}$ mesoscopic model for a sheet of graphene was established by Cranford and Buehler ${ }^{26}$ utilizing coarsegrain bead-spring elements with rotational-spring potentials. This model has been previously implemented to investigate the folding, twisting and coiling of graphene sheets, and the reasonable relationships have been obtained between folded length and bending stiffness, and between strain energy and rotation angle. ${ }^{26,49}$ Also, the fundamental bending mode has been directly observed by Chopin and Kudrolli when the length of graphene ribbon is much larger than its width, ${ }^{50}$ i.e., $L^{\mathrm{f}} \gg W^{\mathrm{f}}$. In the $2 \mathrm{D}$ model, each bond-bead represents a $2.5 \times 2.5 \mathrm{~nm}^{2}$ atomically mono- or multilayer graphene sheets (Figure 1b-III). A harmonic spring potential

$$
\phi_{T}=k_{T}\left(r-r_{0}\right)^{2} / 2
$$

was used to describe the axial stretching energy among all pairs of bonded beads, where $k_{T}$ is the spring constant and $r$ is the distance between two beads with a referenced equilibrium distance $r_{0}=2.5$ $\mathrm{nm}$. A harmonic rotational-spring potential

$$
\phi_{\varphi}=k_{\varphi}\left(\varphi-\varphi_{0}\right)^{2} / 2
$$


was used to describe the in-plane bending energy under shear deformation, where $k_{\varphi}$ is the spring constant related to the bending angle $\varphi$ among the three bond-beads with a referenced equilibrium angle $\varphi_{0}=90^{\circ}$. Another harmonic rotational-spring potential

$$
\phi_{\theta}=k_{\theta}\left(\theta-\theta_{0}\right)^{2} / 2
$$

was used to describe the out-of-plane bending energy with a spring constant $k_{\theta}$, where $\theta$ is the bending angle among the three bond-beads with a referenced value $\theta_{0}=180^{\circ}$. The intralayer vdW interaction between different beads in an in-plane flake was described as a Lennard-Jones potential

$$
\phi_{\text {ip }}=4 \varepsilon_{\text {ip }}\left[\left(\sigma_{\mathrm{ip}} / r\right)^{12}-\left(\sigma_{\mathrm{ip}} / r\right)^{6}\right]
$$

where $\varepsilon_{\text {ip }}$ is an energy-scale parameter determining the depth of potential well, $\sigma_{\text {ip }}$ is a length-scale parameter that determines the position of minimum potential, and $r$ is the bead-to-bead distance in a cutoff range within the in-plane flake. The energy conservation between atomistic and mesoscale models was enforced to match the model parameters listed in Table S1 in Supporting Information, through elastic strain energy by incorporating normal and shear strains, out-of-plane bending, and intramolecular interactions.

Fabrication of 3D GF Numerical Sample. As for the preprocessing initial GF geometrical configuration, all flakes with randomly normal vectors were placed in a huge cubic space lattice to ensure no mutual contact among them. Then, every square flake in the huge cube would be dug an $S=L^{\mathrm{h}} \times W^{\mathrm{h}} \mathrm{nm}^{2}$ hole to ensure an initial multiporous prototype with intrinsic-hole graphene sheets. The NPT assemble technique with a constant temperature of $300 \mathrm{~K}$ and ten barometric pressures (10 bar) was used to deal with the huge cubic numerical GF system with a periodic boundary condition in three directions. The system shrank gradually and finally reached a pre-equilibrium state at $\sim 50 \mathrm{~ns}$ with a time step of $1.0 \mathrm{fs}$. Subsequently, by resetting the Berendsen barostat to $1.0 \mathrm{~atm}$ and the Langevin thermostat to $300 \mathrm{~K}$, the system expanded slightly and reached an equilibrium state at $\sim 80 \mathrm{~ns}$ with the criterion that the total energy fluctuation converges to less than $1 \%$. The obtained numerical GF sample was filled by approximately uniformly distributed holeflakes. On this basis, the 3D linkages were added into the relaxed sample among different hole-flakes at a distance of $\sim 2.51 \mathrm{~nm}$. The number density of the cross-links is defined as a number ratio of the cross-links to bead-bonds, i.e., $\rho_{n}=n_{\mathrm{cl}} / n_{\mathrm{bd}}$, which was set as $\sim 0.068$ for all the GF samples. Finally, the system with linkages and holesheets was minimized with stopping tolerance for force of $1.0 \times 10^{-10}$ $\mathrm{kcal} / \mathrm{mol} / \mathrm{nm}$ to reach its final configuration. The fabricated GF with intrinsic-hole flakes has a cubic length from $\sim 170$ to $\sim 190 \mathrm{~nm}$ with a mass density from $\sim 137$ to $\sim 387 \mathrm{mg} / \mathrm{cm}^{3}$, as shown in Figure 1a.

Uniaxial Supercompression and Uncompression. To gain an in-depth understanding of the typical two-stage supercompression stress-strain relationship and the corresponding time-dependent recovery behavior, a series of uniaxial supercompression and uncompression in deformation-controlled loading and pressurecontrolled unloading-holding were conducted on our numerical GF models. The static equilibrium of the GF network with intrinsic-hole graphene-flakes was achieved by the conjugate gradient method. The compressive deformation rate was set as $10 \mathrm{~nm} / \mathrm{ns}$ along the $x$ direction (Figure 2a) at room temperature using a zero-pressure barostat in other two directions, compressing the sample with a time step of 1.0 fs until the strain between $91 \%$ and $95 \%$. Subsequently, resetting the Berendsen barostat to zero atmosphere along the $x$ direction and keeping other thermal/mechanical conditions unchanged uncompressed the GF system. On this basis, the holding time was set as at least $80 \mathrm{~ns}$ to simulate the time-dependent recovery behavior, which can be captured for kinetic studies with high spatiotemporal resolution in the practical application of GFs, by means of ultrafast pump and probe techniques. ${ }^{52,53}$ The transverse/ longitudinal strain is defined as

$$
\varepsilon_{h}^{m}=\left(l_{h}-l_{h 0}\right) / l_{h 0} \quad(h=x, y, z)
$$

where $l_{h}$ and $l_{h 0}$ are the starting and current length of the sample in the deformation direction, respectively. The definitions of Poisson ratios are defined as the negative ratios between the transverse and the longitudinal deformations for two different nonloading directions, respectively, i.e.,

$$
v_{x y}=-\frac{\varepsilon_{y}^{m}}{\varepsilon_{x}^{m}} \quad \text { and } \quad v_{x z}=-\frac{\varepsilon_{z}^{m}}{\varepsilon_{x}^{m}}
$$

All the simulations were implemented using an open-source software Large-scale Atomic/Molecular Massively Parallel Simulator (LAMMPS) ${ }^{54}$ at Multiscale Computing Hub of Graphene-like-based Materials (MHM) and Supercomputing Center of Lv Liang Cloud Computing Center in China.

Local (Position-Dependent) State of Stress. On the nano-/ mesoscale, the virial stress can be viewed as a measure of the mechanical stress, even when simulating very inhomogeneous phenomena. ${ }^{55}$ On the basis of a generalization of the virial theorem, the average virial stress over a volume $\Omega_{\mathrm{i}}$ around a particle $i$ at a position $\mathbf{r}_{i}$ can be expressed as ${ }^{56}$

$$
\boldsymbol{\sigma}^{i}=\frac{1}{\Omega_{i}}\left(-m \frac{\mathrm{d} \mathbf{u}_{i}}{\mathrm{~d} t} \otimes \frac{\mathrm{d} \mathbf{u}_{i}}{\mathrm{~d} t}+\frac{1}{2} \sum_{j(\neq i)} \mathbf{r}_{i j} \otimes \mathbf{f}_{i j}\right)
$$

where $m$ is the mass of particle in the GF numerical sample. $\mathbf{u}_{i}$ is the displacement of $i$ relative to the reference position; therefore, its material time derivative is the thermal excitation velocity of particle. The interparticle force $\mathbf{f}_{i j}$ applied on the particle $i$ by the particle $j$ can be expressed as

$$
\mathbf{f}_{i j}=\frac{\partial \Phi_{i j}}{\partial \mathbf{r}_{i j}} \frac{\mathbf{r}_{i j}}{\left\|\mathbf{r}_{i j}\right\|} \quad \text { with } \quad \mathbf{r}_{i j}=\mathbf{r}_{i}-\mathbf{r}_{j}
$$

where $\Phi_{i j}$ is the energy of coarse-grain group in consideration, dating from the bead-spring and rotational-spring as well as pairwise interatomic potentials, as reported previously. The equivalence of virial stress and Cauchy stress was reviewed by Subramaniyan and Sun using both theoretical arguments and numerical simulations. ${ }^{57}$ It is noted that besides the flake's deformation, the decomposed rigid translation and rotation of the building graphene blocks both contribute to the atomic stress because they connect with the interatomic distances and thus the potential term of virial stress. The compressive stress is given by a symmetric pressure tensor of the entire system, stored as a 6-element vector, i.e., $\left\{\sigma^{m}\right\}=\left[\sigma_{x x}^{m} \sigma_{y y}^{m} \sigma_{z z}^{m} \sigma_{x y}^{m}\right.$ $\left.\sigma_{y z}^{m} \sigma_{x z}^{m}\right]^{T}$, which is defined as the ensemble average of negative of stress tensor

$$
\boldsymbol{\sigma}^{m}(t)=-\frac{\left\langle\Omega_{i} \boldsymbol{\sigma}^{i}(t)\right\rangle}{V} \quad \text { with } \quad V=\sum_{i=1}^{N} \Omega_{i}
$$

where $V$ is the volume of whole GF sample, and $N$ is the number of total particles involved.

\section{ASSOCIATED CONTENT}

\section{Supporting Information}

The Supporting Information is available free of charge on the ACS Publications website at DOI: 10.1021/acsnano.8b06558.

Force field and geometry parameters, supplementary mechanical behaviors and further discussions, as well as a list of auxiliary videos (PDF)

Movie S1 (AVI)

Movie S2 (AVI)

Movie S3 (AVI)

Movie S4 (AVI)

Movie S5 (AVI) 


\section{AUTHOR INFORMATION}

\section{Corresponding Authors}

*E-mail: pandx@lnm.imech.ac.cn.

*E-mail: xjwang@iamt.ac.cn.

\section{ORCID}

Douxing Pan: 0000-0002-9177-134X

\section{Author Contributions}

D.P. launched the project, carried out all the programming and simulating work, and wrote the paper. C.W. was involved in codesigning the study. X.W. coordinated and supervised the study. All of the authors contributed to discussions.

\section{Notes}

The authors declare no competing financial interest.

\section{ACKNOWLEDGMENTS}

D.P. is thankful for support from the National Natural Science Foundation for the Youth (NSFY) of China (Grant No. 11802306) for the project of the mechanical behavior and corresponding physical essence of the graphene foam on the mesoscale. The study was also supported by the National Natural Science Foundation for the General Program (NSFG) of China (Grant No. 11572320), and Chongqing City Basic and Frontier Research Project (cstc2015jcyjBX0135). The authors are thankful to Ms. Jialu Geng for processing a part of the datum and Mr. Ling Gong for helpful academic exchange.

\section{REFERENCES}

(1) Wang, J.; Ellsworth, M. W. Graphene aerogels. ECS Trans 2009, 19, 241-247.

(2) Wu, Z. S.; Sun, Y.; Tan, Y. Z.; Yang, S.; Feng, X.; Müllen, K. Three-Dimensional Graphene-Based Macro- and Mesoporous Frameworks for High-Performance Electrochemical Capacitive Energy Storage. J. Am. Chem. Soc. 2012, 134, 19532-19535.

(3) Fang, Q. L.; Shen, Y.; Chen, B. L. Synthesis, Decoration and Properties of Three-Dimensional Graphene-Based Macrostructures: A review. Chem. Eng. J. 2015, 264, 753-771.

(4) Idowu, A.; Boesl, B.; Agarwal, A. 3D Graphene Foam-Reinforced Polymer Composites-A Review. Carbon 2018, 135, 52-71.

(5) Qiu, L.; Huang, B.; He, Z. J.; Wang, Y. Y.; Tian, Z. M.; Liu, J. Z.; Wang, K.; Song, J. C.; Gengenbach, T. R.; Li, D. Extremely Low Density and Super-Compressible Graphene Cellular Materials. Adv. Mater. 2017, 29, 1701553.

(6) Tao, Y.; Xie, X. Y.; Lv, W.; Tang, D. M.; Kong, D. B.; Huang, Z. H.; Nishihara, H.; Ishii, T.; Li, B. H.; Golberg, D.; Kang, F. Y.; Kyotani, T.; Yang, Q. H. Towards Ultrahigh Volumetric Capacitance: Graphene Derived Highly Dense but Porous Carbons for Supercapacitors. Sci. Rep. 2013, 3, 2975.

(7) Huang, H.; Chen, P. W.; Zhang, X. T.; Lu, Y.; Zhan, W. C. Edgeto-Edge Assembled Graphene Oxide Aerogels with Outstanding Mechanical Performance and Superhigh Chemical Activity. Small 2013, 9, 1397-1404.

(8) Xu, X.; Zhang, Q.; Yu, Y.; Chen, W.; Hu, H.; Li, H. Naturally Dried Graphene Aerogels with Superelasticity and Tunable Poisson's Ratio. Adv. Mater. 2016, 28, 9223-9230.

(9) Zhang, Q. Q.; Xu, X.; Lin, D.; Chen, W. L.; Xiong, G. P.; Yu, Y. K.; Fisher, T. S.; Li, H. Hyperbolically Patterned 3D Graphene Metamaterial with Negative Poisson's Ratio and Superelasticity. Adv. Mater. 2016, 28, 2229-2237.

(10) Qiu, L.; Liu, J. Z.; Chang, S. L. Y.; Wu, Y.; Li, D. Biomimetic Superelastic Graphene-Based Cellular Monoliths. Nat. Commun. 2012, 3, 1-7.

(11) Wu, Y. P.; Yi, N. B.; Huang, L.; Zhang, T. F.; Fang, S. L.; Chang, H. C.; Li, N.; Oh, J.; Lee, J. A.; Kozlov, M.; Chipara, A. C.; Terrones, H.; Xiao, P. S.; Long, G. K.; Huang, Y.; Zhang, F.; Zhang, L.; Lepró, X.; Haines, C.; Lima, M. D.; et al. Three-Dimensionally
Bonded Spongy Graphene Material with Super Compressive Elasticity and Near-Zero Poisson's Ratio. Nat. Commun. 2015, 6, 6141.

(12) Nautiyal, P.; Boesl, B.; Agarwal, A. The Mechanics of Energy Dissipation in a Three-Dimensional Graphene Foam with Macroporous Architecture. Carbon 2018, 132, 59-64.

(13) Nautiyal, P.; Boesl, B.; Agarwal, A. Harnessing Three Dimensional Anatomy of Graphene Foam to Induce Superior Damping in Hierarchical Polyimide Nanostructures. Small 2017, 13, 1603473.

(14) Nautiyal, P.; Mujawar, M.; Boesl, B.; Agarwal, A. In Situ Mechanics of 3D Graphene Foam Based Ultra-Stiff and Flexible Metallic Metamaterial. Carbon 2018, 137, 502-510.

(15) Xu, Z.; Zhang, Y.; Li, P.; Gao, C. Strong, Conductive, Lightweight, Neat Graphene Aerogel Fibers with Aligned Pores. ACS Nano 2012, 6, 7103-7113.

(16) Peng, Q. Y.; Li, Y. B.; He, X. D.; Gui, X. C.; Shang, Y. Y.; Wang, C. H.; Wang, C.; Zhao, W. Q.; Du, S. Y.; Shi, E. Z.; Li, P. X.; Wu, D. H.; Cao, A. Y. Graphene Nanoribbon Aerogels Unzipped from Carbon Nanotube Sponges. Adv. Mater. 2014, 26, 3241-3247.

(17) Nieto, A.; Boesl, B.; Agarwal, A. Multiscale Intrinsic Deformation Mechanisms of 3D Graphene Foam. Carbon 2015, 85, 299-308.

(18) Krueger, E.; Chang, A. N.; Brown, D.; Eixenberger, J.; Brown, R.; Rastegar, S.; Yocham, K. M.; Cantley, K. D.; Estrada, D. Graphene Foam as a Three-Dimensional Platform for Myotube Growth. ACS Biomater. Sci. Eng. 2016, 2, 1234-1241.

(19) Nieto, A.; Dua, R.; Zhang, C.; Boesl, B.; Ramaswamy, S.; Agarwal, A. Three Dimensional Graphene Foam/Polymer Hybrid as a High Strength Biocompatible Scaffold. Adv. Funct. Mater. 2015, 25, 3916-3924.

(20) Baimova, A.; Korznikova, E. A.; Dmitriev, S. V.; Liu, B.; Zhou, K. Review on Crumpled Graphene: Unique Mechanical Properties. Rev. Adv. Mater. Sci. 2014, 39, 69-83.

(21) Baimova, J.; Rysaeva, L. K.; Liu, B.; Dmitriev, S. V.; Zhou, K. From Flat Graphene to Bulk Carbon Nanostructures. Phys. Status Solidi B 2015, 252, 1502-1507.

(22) Wang, C.; Zhang, C.; Chen, S. H. The Microscopic Deformation Mechanism of 3D Graphene Foam Materials under Uniaxial Compression. Carbon 2016, 109, 666-672.

(23) Wang, C.; Pan, D. X.; Chen, S. H. Energy Dissipative Mechanism of Graphene Foam Materials. Carbon 2018, 132, 641-50.

(24) Qin, Z.; Jung, G. S.; Kang, M. J.; Buehler, M. J. The Mechanics and Design of a Lightweight Three-Dimensional Graphene Assembly. Sci. Adv. 2017, 3, 1601536.

(25) Pan, D. X.; Wang, C.; Wang, T. C.; Yao, Y. G. Graphene Foam: Uniaxial Tension Behavior and Fracture Mode Based on a Mesoscopic Model. ACS Nano 2017, 11, 8988-8997.

(26) Cranford, S.; Buehler, M. J. Twisted and Coiled Ultralong Multilayer Graphene Ribbons. Modell. Simul. Mater. Sci. Eng. 2011, $19,054003$.

(27) Ruiz, L.; Xia, W.; Meng, Z.; Keten, S. A Coarse-Grained Model for the Mechanical Behavior of Multi-Layer Graphene. Carbon 2015, $82,103-115$.

(28) Shen, Z.; Ye, H.; Zhou, C.; Kröger, M.; Li, Y. Size of Graphene Sheets Determines the Structural and Mechanical Properties of 3D Graphene Foams. Nanotechnology 2018, 29, 104001.

(29) Xia, W. J.; Vargas Lara, F.; Keten, S.; Douglas, J. F. Structure and Dynamics of a Graphene Melt. ACS Nano 2018, 12, 5427-5435.

(30) Christensen, R. M. Theory of Viscoelasticity; Dover Publications: New York, 2010.

(31) Jiang, H.; Zhang, J. W.; Kang, G. Z.; Xi, C. C.; Jiang, C. K.; Liu, Y. J. A Test Procedure for Separating Viscous Recovery and Accumulated Unrecoverable Deformation of Polymer under Cyclic Loading. Polym. Test. 2013, 32, 1445-1451.

(32) Chae, S. K.; Gunes, F.; Kim, K. K.; Kim, E. S.; Han, G. H.; Kim, S. M.; Shin, H.-J.; Yoon, S.-M.; Choi, J.-Y.; Park, M. H.; Yang, C. W.; Didier Pribat, D.; Lee, Y. H. Synthesis of Large-Area Graphene Layers on Poly-Nickel Substrate by Chemical Vapor Deposition: Wrinkle Formation. Adv. Mater. 2009, 21, 2328-2333. 
(33) Xu, X.; Li, H.; Zhang, Q. Q.; Hu, H.; Zhao, Z. B.; Li, J. H.; Li, J. Y.; Qiao, Y.; Gogotsi, Y. Self-Sensing, Ultralight, and Conductive 3D Graphene/Iron Oxide Aerogel Elastomer Deformable in a Magnetic Field. ACS Nano 2015, 9, 3969-3977.

(34) Sun, H.; Xu, Z.; Gao, C. Multifunctional, Ultra-Flyweight, Synergistically Assembled Carbon Aerogels. Adv. Mater. 2013, 25, 2554-2560.

(35) Banhart, F.; Kotakoski, J.; Krasheninnikov, A. V. Structural Defects in Graphene. ACS Nano 2011, 5, 26-41.

(36) Liu, X. F.; Zhang, Z. H.; Guo, W. L. Universal Rule on Chirality-Dependent Bandgaps in Graphene Antidot Lattices. Small 2013, 9, 1405-1410.

(37) Chen, H.; Müller, M. B.; Gilmore, K. J.; Wallace, G. G.; Li, D. Mechanically Strong, Electrically Conductive, and Biocompatible Graphene Paper. Adv. Mater. 2008, 20, 3557-3561.

(38) Liu, F.; Wang, C.; Tang, Q. Conductivity Maximum in 3D Graphene Foams. Small 2018, 14, 381801458.

(39) Worsley, M. A.; Pauzauskie, P. J.; Olson, T. Y.; Biener, J.; Satcher, J. H., Jr; Baumann, T. F. Synthesis of Graphene Aerogel with High Electrical Conductivity. J. Am. Chem. Soc. 2010, 132, 1406714069.

(40) Kachanov, L. M. Fundamentals of the Theory of Plasticity; Dover Publications: New York, 2004.

(41) Pan, D. X.; Kang, G. Z.; Zhu, Z. W.; Liu, Y. J. Experimental Study on Uniaxial Time-Dependent Ratcheting of a Polyetherimide Polymer. J. Zhejiang Univ., Sci., A 2010, 11, 804-810.

(42) Reddy, S. K.; Ferry, D. B.; Misra, A. Highly Compressible Behavior of Polymer Mediated Three-Dimensional Network of Graphene Foam. RSC Adv. 2014, 4, 50074-50080.

(43) Zhang, Q. Q.; Zhang, F.; Medarametla, S. P.; Li, H.; Zhou, C.; Lin, D. 3D Printing of Graphene Aerogels. Small 2016, 12, 17021708.

(44) Lee, C.; Wei, X.; Kysar, J. W.; Hone, J. Measurement of the Elastic Properties and Intrinsic Strength of Monolayer Graphene. Science 2008, 321, 385-388.

(45) Yang, S. Y.; O’Cearbhaill, E. D.; Sisk, G. C.; Park, K. M.; Cho, W. K.; Villiger, M.; Bouma, E. B.; Pomahac, B.; Karp, J. M. A Bioinspired Swellable Microneedle Adhesive for Mechanical Interlocking with Tissue. Nat. Commun. 2013, 4, 1702.

(46) Waduge, P.; Larkin, J.; Upmanyu, M.; Kar, S.; Wanunu, M. Programmed Synthesis of Freestanding Graphene Nanomembrane Arrays. Small 2015, 11, 597-603.

(47) Zhao, Y.; Liu, J.; Hu, Y.; Cheng, H. H.; Hu, C. G.; Jiang, C. C.; Jiang, L.; Cao, A. Y.; Qu, L. T. Highly Compression-Tolerant Supercapacitor Based on Polypyrrole-Mediated Graphene Foam Electrodes. Adv. Mater. 2013, 25, 591-595.

(48) Newnham, R. E.; Skinner, D. P.; Cross, L. E. Connectivity and Piezoelectric-Pyroelectric Composites. Mater. Res. Bull. 1978, 13, $525-536$.

(49) Cranford, S.; Sen, D.; Buehler, M. J. Meso-Origami: Folding Multilayer Graphene Sheets. Appl. Phys. Lett. 2009, 95, 123121.

(50) Chopin, J.; Kudrolli, A. Helicoids, Wrinkles, and Loops in Twisted Ribbons. Phys. Rev. Lett. 2013, 111, 174302.

(51) Cabosart, D.; Felten, A.; Reckinger, N.; Iordanescu, A.; Toussaint, S.; Faniel, S.; Hackens, B. Recurrent Quantum Scars in a Mesoscopic Graphene Ring. Nano Lett. 2017, 17, 1344-1349.

(52) King, W. E.; Campbell, G. H.; Frank, A.; Reed, B.; Schmerge, J. F.; Siwick, B. J.; Stuart, B. C.; Weber, P. M. Ultrafast Electron Microscopy in Materials Science, Biology, and Chemistry. J. Appl. Phys. 2005, 97, 8.

(53) Plemmons, D. A.; Suri, P. K.; Flannigan, D. J. Probing Structural and Electronic Dynamics with Ultrafast Electron Microscopy. Chem. Mater. 2015, 27, 3178-3192.

(54) Plimpton, S. Fast Parallel Algorithms for Short-Range Molecular Dynamics. J. Comput. Phys. 1995, 117, 1-19.

(55) Hardy, R. J. Formulas for Determining Local Properties in Molecular Dynamics Simulations: Shock Waves. J. Chem. Phys. 1982, $76,622-628$.
(56) Swenson, R. J. Comments on Virial Theorems for Bounded Systems. Am. J. Phys. 1983, 51, 940-942.

(57) Subramaniyan, A. K.; Sun, C. T. Continuum Interpretation of Virial Stress in Molecular Simulations. Int. J. Solids Struct. 2008, 45, 4340-4346. 